\title{
The Declaration to Improve Biomedical \& Health Research
}

It is extraordinary that we tolerate a situation in which most research activity is wasted. ${ }^{1}$ Research findings are mostly wrong ${ }^{2}$ and riddled with biases including inadequate or absent reporting ${ }^{3}$ and hidden conflicts of interest ${ }^{4}$. Since Doug Altman blew the whistle on this scandal over 25 years ago there has been growing acceptance of the scale of the problem and the need for change. ${ }^{56}$ But specific actions must follow.

We are an international group of researchers and patients who believe that:

- it is ethically untenable to remain complicit in the crises that undermine science,

- there are simple measures which can improve the quality and openness, and

- the public and patients have a right to full access of the research they fund and participate in

To start fixing science's problems we need to rally around a specific set of achievable demands. The ideas we present are not new. Nor will they solve all the problems that face medical research. But they are simple measures which could bring rapid and significant improvements.

\section{We demand:}

\section{1) Mandatory registration of interests}

Failure to disclose potential conflicts of interest is widespread. ${ }^{7}$ Conflicting interests do not necessarily invalidate research but their existence has to be known to interpret findings.

Although voluntary registers of interest already exist ${ }^{8}$ uptake is low and the necessity of scouring many possible sources for voluntary disclosure is a barrier to transparency. There must be an expectation that everyone involved in research, from patient groups to researchers, from peer reviewers to funders, declare all their interests in a central and assessable location. This is eminently achievable. In the US, such an obligation already exists for physicians ${ }^{9}$ and there have been calls for the General Medical Council to require the same in the UK ${ }^{10}$.

Over seven million researchers are already listed on the Open Research and Contributor ID (ORCID) system with registration already required by many institutions and journals. ORCID could catalogue declarations of interests with links embedded in publications. While the US experience suggests that legislation might be required to mandate disclosure, in the meantime, academic institutions, journals and funders could begin to work with ORCID to facilitate a comprehensive system of declaration.

\section{2) That journals promote registered reports}

Post-hoc abuses such as 'outcome switching' and so-called p-hacking are rife. The incentive to generate 'interesting' results drives undeclared repeated analyses to fit publishable narratives. Even when deviations from protocols are identified the supposed 'self-correcting' 
mechanism of peer scrutiny simply does not work. ${ }^{11}$ 'Registered reports' are an elegant solution. ${ }^{12}$ Authors submit their methods prior to data collection and analysis and if these satisfy peer review, journals commit to publication, regardless of the results generated. Authors may face greater workload in the planning of studies but benefit from peer review at the earliest stages. Registered reports differ from clinical trial registration ${ }^{13}$ and they are applicable to any hypothesis-driven research, rather than only clinical trials.

As of February 2020 only about $1 \%$ of medical journals allow authors to submit registered reports. ${ }^{14}$ We call on all journals to promote registered reports, with the target that at least $20 \%$ studies in ICMJE journals are published as registered reports by 2025 . We also call on public and charitable bodies to require that all hypothesis driven research they fund is published as registered reports within 10 years.

\section{3) That all publicly funded research is registered and published in designated Research Repositories}

The majority of research is funded by public and charitable funds. Yet, huge amounts of research is never published at all, which aside from being an indefensible waste of public money, is a major source of publication bias ${ }^{3}$. Meanwhile, basic research documentation which is essential to ensure appropriate research conduct, such as protocols, are only sometimes available, either on voluntary databases or upon agreement of study authors.

The World Health Organization (WHO) has long urged registration of trials in affiliated 'primary registries', such as ClinicalTrials.gov ${ }^{15}$ and the EU Clinical Trials Register ${ }^{16}$ which can all be searched simultaneously through a dedicated WHO website ${ }^{17}$. Mandatory registration of trials has improved transparency, although compliance with publication requirements is poor ${ }^{18}$, possibly hampered by problems with the basic functionality of some major registries ${ }^{19}$. Even where trials have been registered, usually only very limited information is shared, rather than the full protocols required to really understand study plans.

Most researchers don't work in trials. Some principled scientists do register their work but while this remains voluntary such researchers are likely to remain in the minority. For all publicly funded research, not just trials, comprehensive documentation including protocols, statistical analysis plans, statistical analysis code and raw or appropriately de-identified summary data should be available on a single WHO affiliated repository, designated for that purpose by each state or groups of states.

Depositing documentation need not become onerous for researchers and could replace much of the overly bureaucratic reporting currently required for funders and ethics committees. Different solutions may exist in different countries. For example, England's Health Research Authority could develop such a registry ${ }^{20}$, by building on its existing public databases ${ }^{21}$. Or, through additional national funding and international support, existing platforms which promote transparency and accessibility 222324 could be designated for this purpose through collaboration with national research bodies. 


\section{Conclusions}

While basic solutions exist remaining complicity with the scandal of poor medical research is not defensible. The solutions we propose will not solve all of the problems, but we think they will be a start. Once they are achieved, further research will be vital to determine the effectiveness of these measures and to identify and remedy any unintended consequences.

We invite you to join the campaign to improve science. Please consider signing the declaration, or help us to develop the declaration by telling us how to improve it.

\section{Find out more}

To sign the declaration or to find out more including a full list of signatories visit us on www.improvehealthresearch.com. To see interests declared by signatories please refer to 'interests' document on our Open Science Framework page https://osf.io/k3w7m 


\section{References}

1. Chalmers I, Glasziou P. Avoidable waste in the production and reporting of research evidence. The Lancet 2009;374(9683):86-89. doi: https://doi.org/10.1016/S0140$\underline{6736(09) 60329-9}$

2. loannidis JPA. Why Most Published Research Findings Are False. PLOS Medicine 2005;2(8):e124. doi: 10.1371/journal.pmed.0020124

3. DeVito NJ, Goldacre B. Catalogue of bias: publication bias. BMJ Evidence-Based Medicine 2019;24(2):53-54. doi: 10.1136/bmjebm-2018-111107

4. Moynihan R, Macdonald $\mathrm{H}$, Heneghan $\mathrm{C}$, et al. Commercial interests, transparency, and independence: a call for submissions. BMJ 2019;365:I1706. doi: 10.1136/bmj.I1706

5. Altman DG. The scandal of poor medical research. BMJ 1994;308(6924):283-84. doi: 10.1136/bmj.308.6924.283

6. Goldacre B, Heneghan C. How medicine is broken, and how we can fix it. BMJ : British Medical Journal 2015;350:h3397. doi: 10.1136/bmj.h3397

7. Saleh RR, Majeed H, Tibau A, et al. Undisclosed financial conflicts of interest among authors of American Society of Clinical Oncology clinical practice guidelines. Cancer;0(0) doi: 10.1002/cncr.32408

8. Kmietowicz Z. Disclosure UK website gives "illusion of transparency," says Goldacre. BMJ 2016;354:i3760. doi: 10.1136/bmj.i3760

9. Silverman E. Everything you need to know about the Sunshine Act. BMJ : British Medical Journal 2013;347:f4704. doi: 10.1136/bmj.f4704

10. McCartney M, Goldacre B, Chalmers I, et al. Why the GMC should set up a central registry of doctors' competing interests. 2014;348:g236. doi: 10.1136/bmj.g236 \%J BMJ : British Medical Journal

11. Goldacre B, Drysdale H, Dale A, et al. COMPare: a prospective cohort study correcting and monitoring 58 misreported trials in real time. Trials 2019;20(1):118. doi: 10.1186/s13063-019-3173-2

12. In praise of process. Nature 2019;571 doi: 10.1038/d41586-019-02277-1

13. Centre for Open Science [Available from: https://cos.io/rr/ accessed 14th September 20192019.

14. Bradley SH, DeVito NJ, Lloyd K, et al. Reducing bias and improving transparency in biomedical and health research: A critical overview of the problems, progress so far and suggested next steps (preprint) 2020.

15. ClinicalTrials.gov: U.S. National Library of Medicine; [Available from: https://www.clinicaltrials.gov/ accessed 25 Jul 2019.

16. EU Clinical Trials Register [Available from: https://www.clinicaltrialsregister.eu/ accessed 18 Nov 2019.

17. International Clinical Trials Registry Platform: World Health Organisation; [Available from: https://www.who.int/ictrp/en/ accessed 18 Nov 2019. 
18. Goldacre B, DeVito NJ, Heneghan C, et al. Compliance with requirement to report results on the EU Clinical Trials Register: cohort study and web resource. BMJ 2018;362:k3218. doi: 10.1136/bmj.k3218

19. Achieving Excellence in Clinical Trials Reporting: Transparimed; [Available from: https://www.transparimed.org/single-post/2019/04/27/Achieving-excellence-in-clinicaltrial-reporting-Tips-and-tricks-from-the-University-of-Nottingham accessed $18 \mathrm{Nov}$ 2019.

20. Make it Public - new consultation launches to help increase public access to research findings: Health Research Authority; 2019 [updated 17 Jun 2019. Available from: https://www.hra.nhs.uk/about-us/news-updates/make-it-public-new-consultationlaunches-help-increase-public-access-research-findings accessed 18 Nov 2019.

21. Health Research Authority, research summaries [Available from:

https://www.hra.nhs.uk/planning-and-improving-research/applicationsummaries/research-summaries/ accessed 14th September 20192019.

22. Open Science Framework [Available from: https://osf.io accessed 18 Nov 2019.

23. Dryad [Available from: https://datadryad.org/stash accessed 18 Nov 2019.

24. Figshare [Available from: https://figshare.com/ accessed 18 Nov 2019. 